\title{
Primer on the Science of In Vitro Dissolution Testing of Oral Dosage Forms and Factors Influencing its Biological Relevance
}

\author{
Raafat Fahmy and Marilyn N. Martinez*
}

Office of New Animal Drug Evaluation, Center for Veterinary Medicine, U.S. Food and Drug Administration, Rockville, MD, USA

e-mail: marilyn.martinez@fda.hhs.gov

\begin{abstract}
In vitro dissolution testing can serve as an effective and efficient tool for evaluating the influence of formulation and manufacturing variables on drug release characteristics. The targeted purpose will determine the method used and the implications of the test results. Regardless of its intended purpose, it is necessary to understand the factors influencing in vitro dissolution outcomes to avoid the introduction of error into the data interpretation. This will influence the selection of the in vitro test procedure, ensuring that the method can identify changes in the critical formulation and manufacturing variables. There is also a need to understand the rate limiting factors influencing in vivo product bioavailability both from a dissolution and an absorption perspective. Finally, to achieve in vivo biorelevance, it is necessary to understand how the selected in vitro dissolution test method reflects the variables impacting in vivo product dissolution behavior. This primer summarizes and integrates these diverse variables, fostering an appreciation of the strengths and potential pitfalls that may be encountered during the generation and analysis of in vitro dissolution profiles associated with oral dosage forms. This understanding is needed for developing a well-designed dissolution test procedure that is appropriately fit for purpose.
\end{abstract}

KEYWORDS: In vitro dissolution, formulation effects, oral drug absorption, in vivo-in vitro relationships

\section{BACKGROUND}

W hether designing in vitro dissolution studies or analyzing the resulting data, it is necessary to appreciate the interrelationship between in vitro product performance, drug physicochemical properties, productformulation, productinvivodissolution behavior, and the biological variables influencing oral drug absorption. In turn, the nature of these relationships are defined by the thermodynamic (equilibrium) solubility of the active pharmaceutical ingredient (API), its ability to move across a biological membrane (enterocyte or the liver), and the fraction of the dose that successfully moves into the systemic circulation. Furthermore, some highly lipophilic compounds and product formulations allow for the preferential absorption of drug into the lymphatic rather than the portal circulation, thereby avoiding first pass metabolism (1-3). These relationships are illustrated in Figure 1.

The development of oral dosage forms typically focuses on solubilization of the API. The formulation can also exert biological effects that influence systemic availability, including the enhancement of lymphatic absorption, modulation of efflux pumps within the enterocyte, altering presystemic drug metabolism, and changing gastrointestinal (GI) transit time (4-9). For example, presystemic drug metabolism or transporter activity can be altered by the inclusion of antioxidants, such as derivatives of Vitamin $\mathrm{E}(10)$.

Possible mechanisms by which lipid and surfactant formulations can increase oral bioavailability include (11):

1. Solubilization or wetting of the dissolving particle;

2. Prolonging residence within the $\mathrm{Gl}$ tract due to decrease in gastric emptying and intestinal motility;

3. Protection against luminal drug degradation;

4. Protection from brush border metabolism;

5. Enhanced membrane permeability;

* Corresponding author. 


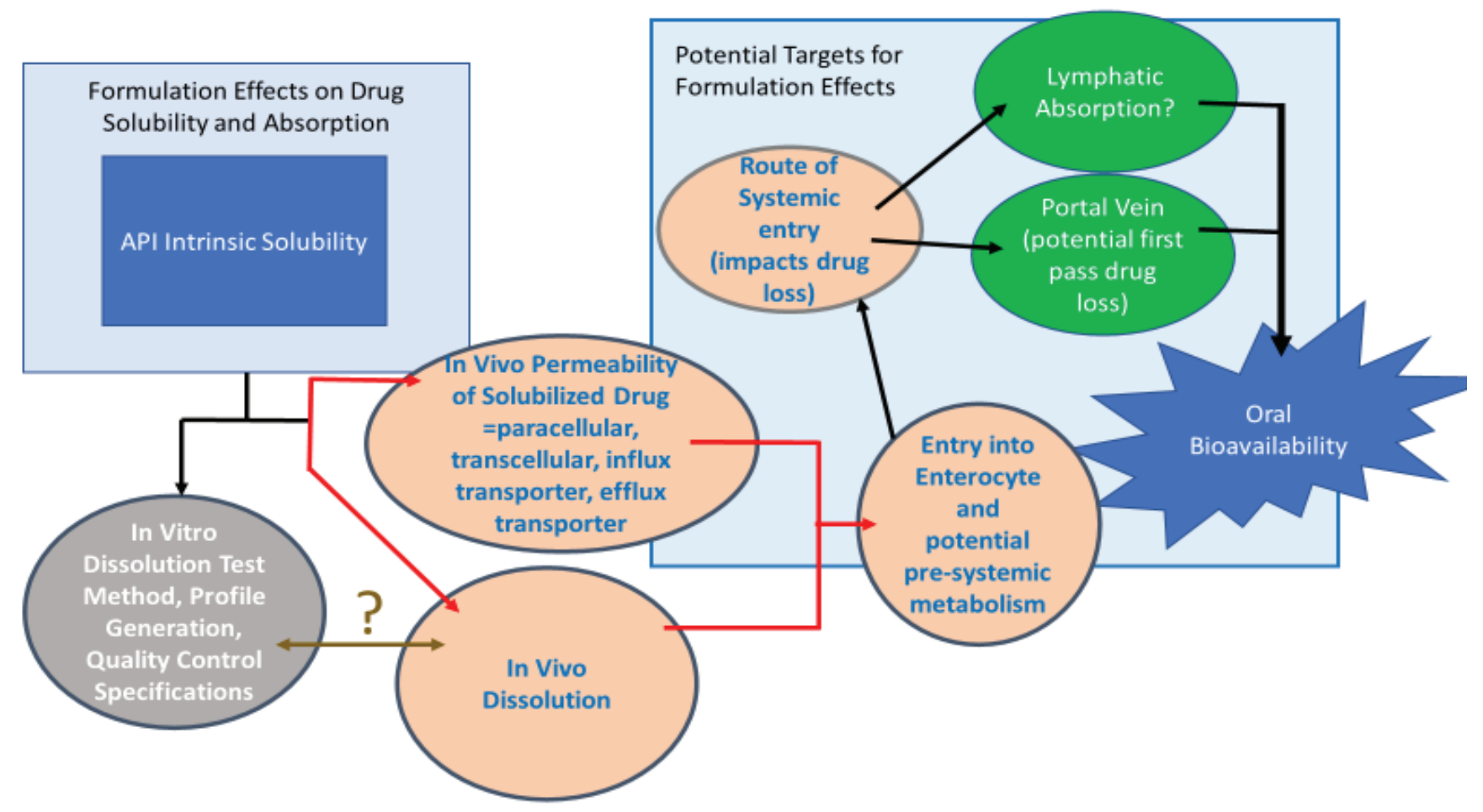

Figure 1. Interrelationship between the active pharmaceutical ingredient (API), formulation, physiology, and bioavailability. The question mark indicates the uncertainty about the relationship between the in vivo and in vitro dissolution profiles. This is a part of the method development that should be considered.

6. Increased membrane contact, which can increase the magnitude of intestinal drug absorption; and

7. Absorption via the lymphatics.

A brief description of additional ways that formulation may influence in vivo drug absorption is provided in the Appendix. Although these points should be considered during efforts to describe an in vivo-in vitro relationship, for this review, only those formulation effects associated with drug solubilization and dissolution will be considered.

\section{UNDERSTANDING THE RELATIONSHIP BETWEEN SOLUBILITY, FORMULATION, AND PRODUCT DISSOLUTION CHARACTERISTICS Defining Solubility}

Various definitions of solubility are described in the proposed new USP general chapter, <1236> (12). The following terms are of importance to the discussions in this review.

Thermodynamic (equilibrium) solubility: Maximum quantity of that substance that can be completely dissolved at a given temperature and pressure in a given amount of solvent and is thermodynamically valid as long as a solid phase exists in equilibrium with the solution phase. This value can differ as a function of $\mathrm{pH}$, so equilibrium solubility is often described across a range of $\mathrm{pH}$ values.

Intrinsic solubility: Concentration of the unionized molecule in a saturated aqueous solution, existing in a thermodynamic equilibrium at a given temperature, and is determined at a $\mathrm{pH}$ where the drug is fully unionized (13).

Saturation solubility: Maximum amount of drug that can be dissolved in a given volume of fluid. It is often quantified by the dose number $\left(D_{0}\right)$ :

$\mathrm{D}_{0}=\frac{M / V_{0}}{C_{S}}$

where $M$ is the maximum administered dose, $V_{o}$ is the volume of the $\mathrm{Gl}$ fluids, and $C_{s}$ is the intrinsic solubility of the drug (14).

For any compound, $D_{0}$ will differ as a function of gastric volume and maximum administered dose.

Apparent or kinetic solubility: Metastable state of a supersaturated condition, returning to a thermodynamically stable state (with precipitation) after 
a period (seconds to months) (15). Some formulations are specifically designed to support a supersaturated state, maximizing the amount of solubilized drug that interacts with the intestinal membrane (16).

From the perspective of the API, the critical factors influencing the API in vivo solubility include (17):

- Intrinsic solubility

- $\quad$ pKa (the pH at which half of the drug is in its ionized form) if it is a weak acid or weak base

- Solid state characteristics (e.g., crystalline habit [i.e., the crystal characteristic external shape], particle size, solvates/hydrous/anhydrous substances). Anhydrous crystals and amorphic substances are typically more soluble forms of a drug.

- Stereochemistry (enantiomers have same solubility, but there can be differences (positive or negative) in solubility of racemic mixture vs separate enantiomers). Different stereoisomers can also influence the activity (safety and effectiveness), absorption, and metabolism of the compound.

- Salt form: Though salt forms are selected to enhance drug solubility, it is important to consider the milieu within which that form must dissolve. More about this issue is described in the next several paragraphs (common ion effects).

Regardless of whether it is the in vivo or in vitro solubilization of the drug that is being considered, the principles controlling the two processes are comparable. Understanding these processes as they pertain to the drug in question will help determine the most appropriate conditions for testing in vitro dissolution and the formulation to be used for in vivo drug delivery.

\section{UNDERSTANDING THE DRUG SOLUBILIZATION PROCESS (12)}

Considering a simple binary system, dissolution occurs spontaneously when the free energy of the solution is less than the sum of the free energies of the solute and solvent. The solubilization process continues until the free energy difference falls to zero, at which point equilibrium is established between the dissolved and undissolved forms of the compound (defining its solubility under that set of conditions).

The enthalpy change, or heat of solution, is controlled by two opposing interactions. Favoring API solubilization is the interaction between a molecule of the API and that of the solvent. Opposing this process are the cohesive energies of the solute for itself. When the attractive forces of solute for solvent predominate, the result will be an exothermic process and a negative heat of solution (i.e., heat is released). Conversely, when the energy required to break solute-solute bonds and solvent-solvent bonds exceeds the energy released by the solute-solvent interaction, the heat of solution is positive (endothermic), leading to a cooling effect. During in vitro dissolution testing, the constraint of solid state interactions is removed and energy is dispersed in the larger volume of the solution. Thus, with few exceptions, the entropy of solution is positive. Indeed, when the heat of solution is positive, dissolution is said to be entropically driven.

Ultimately, dissolution is an interfacial phenomenon. The surface tensions (surface free energy), which refers to the interface between a condensed phase (i.e., solid or liquid) and a gas, will be a critical determinant of the rate at which phase-interactions can occur. Each phase in the typical dissolution process has its own "surface" tension. For example, water has a relatively high surface tension resulting from strong intermolecular interactions dominated by hydrogen bonding. Water will spontaneously interact with (e.g., wet or spread on) a highenergy surface like cellulose, but it will not spontaneously wet a low energy surface like magnesium stearate. Drugs that are characterized as having low energy surfaces are typically low solubility compounds.

Another consideration pertains to the ability to maintain a concentration gradient favoring drug dissolution. According to the diffusion layer theory, there is a region (or stagnant layer) that exists adjacent to the solid particle. The solution of dissolved drug at the liquid solid interface is saturated, and diffusion from this region of high concentration to bulk solution occurs across the thickness of the boundary layer. The greater the concentration gradient - that is the more dilute the solution - the faster the mass transfer of solute through the boundary layer into the surrounding medium. For this reason, the dissolution rate of an immediate release formulation is typically fastest at the onset of dissolution and decreases as the concentration of the solution increases.

\section{UNDERSTANDING IN VIVO FORMULATION EFFECTS}

In vitro test development and their in vivo biorelevance rely upon product understanding and an appreciation of the rate-limiting factors influencing in vivo product 
performance. Because recently developed APIs often exhibit very strong self-affinity (low aqueous solubility), there is a focus on identifying formulation strategies that reduce the strength of these internal interactions (18). Pharmaceutical techniques for enhancing aqueous solubility include use of solubility-enhancing excipients or molecular modifications (e.g., alternative salt forms, development of amorphous drug substance, or changes in particle size) (19).

For poorly soluble drugs, the problem may not simply be getting the drug into solution but also maintaining this solubilized state. Supersaturation strategies include solvent-cosolvent systems, lipid-based drug delivery, and use of amorphous drug substances (20). Co-crystals and crystalline salt forms can also improve drug solubility and dissolution properties without being thermodynamically unstable. Alternatively, inorganic materials such as silica are being considered as carriers for the delivery of poorly water-soluble drugs (21-23).

\section{IN VITRO DISSOLUTION: POINTS TO CONSIDER}

\section{Basic Considerations}

The intrinsic dissolution rate is defined as the amount of drug substance that goes into solution per unit time under standardized conditions of temperature, $\mathrm{pH}$, and solvent composition and constant surface area (24). Typically, we are more concerned with the dissolution rate of the formulated product than of the pure API. An overview of the history of in vitro dissolution testing was reviewed by Patadia et al. (25). Optimally, the dissolution method should be established with the goal of being both discriminating (being able to identify changes in critical quality attributes of the drug product), biopredictive (predictive of those changes that will influence in vivo product performance), and sensitive to any changes in product integrity during its shelf life.

For any given product, the rate and extent of dissolution is determined by interactions between the solid particle and the liquid phases, solid-liquid mass transfer, rate and extent of particle disintegration and erosion, the ability of the particle to be suspended (as opposed to precipitating) within the dissolving fluids, and the interactions between the particle and the dissolving medium. Because dissolution is a dynamic process, fluid velocity and shear distributions (which can be affected by the location of the tablet within the vessel and the geometry of the system) can have measurable influence in the shape of the in vitro dissolution profile. These points are detailed later in this review.
The shape of the in vitro drug dissolution profile can be influenced by the following variables $(26,27)$.

- Apparatus (note: position of apparatus, shape of vessel, vibrations, and other factors can influence in vitro test results). The considerations of sink conditions and mixing capability and an appreciation of the material constraints imposed by the dosage form are integrated into the selection of the dissolution apparatus (see Appendix for a description of the seven standardized USP apparatuses for dissolution).

- Agitation speed

- $\quad$ Temperature

- Media composition ( $\mathrm{pH}$, ionic composition and strength, solvents, and cosolvents). The selection of $\mathrm{pH}$ reflects whether the API is a weak acid or base, its $\mathrm{pKa}$, and $\mathrm{pH}$-associated changes in API stability. When possible, the $\mathrm{pH}$ selected should provide sink conditions and minimize the need to add a surfactant or solvent.

- Media volume. Typically, the volume of solvent used when conducting in vitro dissolution testing is determined by the equilibrium solubility of the API and the fluid volume needed to maintain sink conditions. The use of sink conditions ensures that the shape of the dissolution profile is influenced primarily by product formulation rather than by API solubility. Sink conditions are defined as no less than three times the volume of fluid needed to have a saturated solution (28). Alternatively, sink conditions can be stated as the volume of fluid needed to fully dissolve three times the targeted amount of drug. However, there are situations when the use of sink conditions may camouflage formulation effects. Failure to discriminate between inequivalent formulations may occur when sink conditions are used to enhance oral bioavailability through the maintenance of a supersaturated state (22).

API solubility and the stability of the API in solution need to be evaluated under several conditions. An Ishikawa diagram that lists all the variables pertaining to APIs and drug product characteristics that could affect the dissolution method development strategy is provided in Figure 2. 


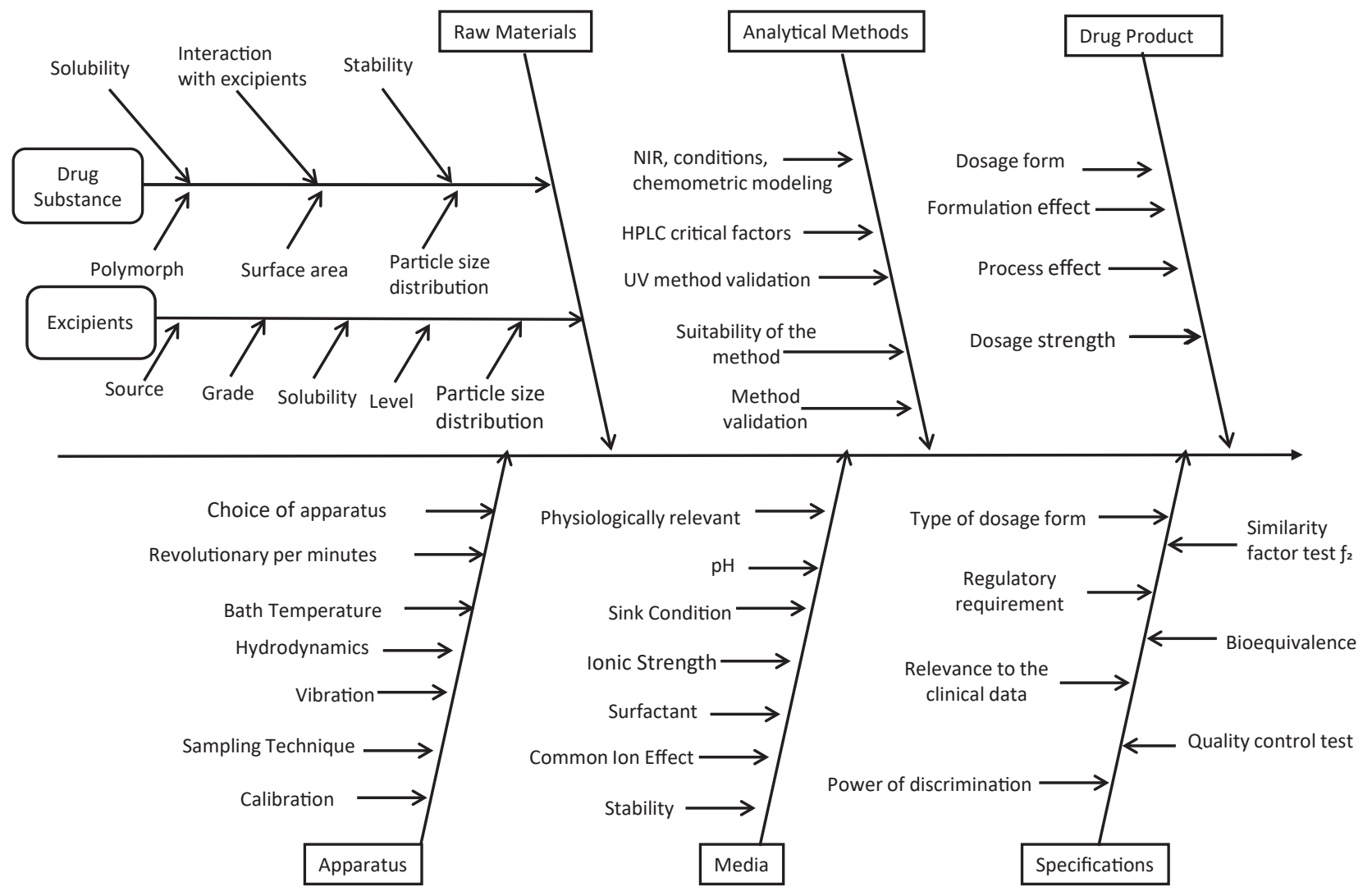

Figure 2. Characteristics of the in vitro dissolution profiles are influenced by the characteristics of the active pharmaceutical ingredient (API), excipients, drug product, and mechanics of the dissolution system, media composition in the dissolution vessel, the analytical methods, and the specifications. NIR, near infrared; HPLC, high-perfomance liquid chromatography; UV, ultraviolet.

\section{DESIGNING THE IN VITRO TEST}

The in vitro test conditions should be developed based on drug physicochemical characteristics, solution stability, and drug-excipient interaction across a range of physiological $\mathrm{pH}$ values. Procedure optimization is contingent on minimizing sources contributing to the variability of the test.

\section{Dissolution Media}

Ideally, dissolution tests should be conducted using a physiologically relevant medium that can be linked to the in vivo performance. The medium should not detract from the ability to discern changes in the critical material attributes or manufacturing variables (quality control purpose). With the goal of achieving sink conditions, the volumes used are frequently within the range of 500-1000 mL (notwithstanding those situations when non-sink conditions are more appropriate, such as for formulations intentionally designed to create a supersaturated state).
To be predictive of in vivo product dissolution, the dissolution media should reflect the critical variables associated with API solubilization. For example, two bioinequivalent formulations of nimodipine (a very poorly soluble drug) were indistinguishable when tested in $0.3 \%$ sodium dodecyl sulfate (SDS) plus acetate buffer $\mathrm{pH} 4.5$, as recommended by the 2000 edition of the British Pharmacopoeia (BP); however, formulation inequivalence was detected when tested in water (29). When the concentration of SDS was lowered to $0.05 \%$, the inequivalence of the two formulations was successfully demonstrated, even though a greater extent of dissolution than that in water was observed.

The pKa of the excipients may also need to be considered. For example, use of a buffered system with a $\mathrm{pH}$ ranging between $\mathrm{pH} 4.5$ and 6.8 can lead to an interaction between the excipient, croscarmellose sodium, and a weak basic drug. For weak bases, it may be appropriate to test in vitro drug release using fasted simulated small intestinal fluid (FaSSIF) and fed simulated small intestinal 
fluid (FeSSIF) media to cover dissolution changes due to food-induced bile salt release and altered $\mathrm{Gl} \mathrm{pH}(30,31)$.

Use of water as a dissolution medium is discouraged because test conditions, such as $\mathrm{pH}$ and surface tension, can differ as a function of the water source. Furthermore, media $\mathrm{pH}$ can drift as the test progresses towards complete product dissolution, thereby necessitating use of a buffer system to control against changes in $\mathrm{pH}$ from the beginning to the end of the test. Buffer selection should be based upon its ability to maintain $\mathrm{pH}$ consistency throughout the test and the absence of a common ion that can suppress the dissociation of a weak electrolyte (32). The presence of a common ion can decrease API ionization and solubilization, thereby favoring the API in its solid form. Alternatively, the presence of an uncommon ion may enhance solubilization if it has a high affinity for the salt form of the API.

In the presence of solubility constraints, there may be a need to employ surfactants. These compounds act as wetting agents by lowering the surface tension between the solid particle and the solvent. At concentrations above the critical micelle concentration, micellar solubilization is promoted. The use of surfactants may be appropriate when the drug is relatively insoluble, regardless of $\mathrm{pH}$, or when solubilization of the API occurs at a nonphysiological (not biologically relevant) $\mathrm{pH}$.

A surfactant is an amphiphilic molecule with polar and nonpolar regions that preferentially concentrate at the solute-solvent interface and decrease the interfacial tension. At higher surfactant concentrations (i.e., above the critical micelle concentration), micelles can be formed that enhance solubility. If a surfactant is needed to maintain sink conditions, its selection should be based on the characteristics of the API (for example, it is not appropriate to use anionic surfactants, such as sodium laurel sulfate, with a cationic drug).

In some cases, there is also a need to add a substance that can enhance the interaction between the solvent and the API by affecting the solvent surface tension. Examples of these dissolution-enhancing cosolvents are dimethyl sulfoxide and propylene glycol. Cosolvents can also positively affect dissolution by interacting with an excipient. For example, the increase in acetylsalicylic acid dissolution from tablets containing aluminum antacid in a medium with $1 \%$ citric acid has been attributed to the complexation of aluminum by the citric acid, thereby providing sink conditions for the excipient. However, in some cases, cosolvents can negatively influence tablet disintegration, thereby counteracting potential positive effects it may have on drug solubility (33). For example, lactose is more soluble in water than it is in alcohol. Therefore, when the dissolution medium contains alcohol, there may be a negative effect on the dissolution of the drug in the formulation, depending upon the amount of lactose in the formulation and the concentration of alcohol (e.g., ethanol or methanol) in the dissolution medium (34).

For capsule formulations, cross-linking of the shell can significantly influence in vitro and in vivo product dissolution. The United States Food and Drug Administration (FDA) Center for Drug Evaluation and Research (CDER) 1997 dissolution guidance (35) indicates the possible need for enzymes (pepsin with SGF and pancreatin with SIF) to dissolve pellicles formed with gelatin capsule products to permit the dissolution of the drug.

\section{Agitation}

The targeted agitation speed should be as low as possible to maintain discriminative power and to reduce the risk of foam development when surfactants are present. Typically, agitation speed for the basket (USP apparatus 1) is maintained at $50-100 \mathrm{rpm}$, and that of the paddle (USP apparatus 2) is $50-75 \mathrm{rpm}$. When these conditions are inadequate to achieve the targeted extent of product dissolution, alternative conditions may need to be considered.

Adjustment of the agitation speed is a mechanism for controlling the fluid hydrodynamics of the test system. By controlling system sheer force, fluid hydrodynamics influence the thickness of the boundary layer, and accordingly, the rate of in vitro dissolution. Hydrodynamics, the motion of fluids and the forces acting on solid bodies, is affected by the velocity and shear stress/strain distribution of the test $(36,37)$. Inadequate control of the system hydrodynamics can introduce variability into the dissolution process $(38,39)$.

\section{System Hydrodynamics}

In addition to agitation speed, system hydrodynamics can be affected by the geometry of the system (vessel shape, agitation method, fluid sampling method) and the configuration of the dissolution apparatus (or tablet location within the dissolution vessel). When failing to achieve well-stirred conditions, there can be regions of high and low mixing and therefore an uneven distribution of dissolved drug. This phenomenon has been extensively studied using USP apparatus 2 (39). 
One of the potential consequences of flawed hydrodynamics is the presence of a coning phenomenon. Coning occurs when the disintegrated mass does not move freely with the surrounding fluid. It is noticeable at typically used paddle rotation speeds such as $50 \mathrm{rpm}$, where a 'dead zone' forms at the bottom of the vessel due to the slower agitation rate, thereby trapping the drug particles. The result is an artificially induced slowing of the product in vitro dissolution rate. Potential solutions this problem include an increase in paddle speed or the use of peak vessels (vessels designed with a convex bottom to disturb building the coning and keep the particles floating into the vessels) $(41,42)$.

\section{METHOD VALIDATION}

Method validation encompasses both the in vitro system and the analytical method, but only system-associated factors will be discussed in this primer. Method validation should demonstrate that the method is scientifically sound and guarantees accuracy, precision, and reproducibility (28).

\section{Setting In Vitro Release Specifications}

Specifications can be used as a tool for: 1) in vivo predictions, to insure consistency between batches; 2) setting age specification; and 3) evaluating product stability throughout the shelf life. Ideally, in vitro specifications should track changes in the critical formulation factors that control the rate and extent of in vivo drug product dissolution. When such in vivo data are not available, specifications should be set based on formulation understanding and an appreciation of drug physicochemical properties.

Depending upon the release profile and duration of the in vitro dissolution and the intended purpose of the in vitro tests, the specifications may necessitate the inclusion of more than a single timepoint. For example, the previously mentioned 1997 FDA CDER dissolution guidance notes that for poorly water soluble drug products, more than one timepoint is recommended for routine quality control to insure consistent in vivo product performance (35). The guidance also remarks that there may be occasions when a complete profile is needed for quality control. Multiple timepoint specifications may also be appropriate for some modified and extended release formulations. Each of these considerations underscores the importance of drug and drug product understanding. In so doing, the specifications can be used not only as a predictor of in vivo product performance, but also for ensuring batchto-batch consistency, identifying changes as a function of age, tablet hardness, moisture content, or excipient changes that can influence in vitro and in vivo product behavior.

\section{Enhancing the Likelihood of In Vitro-In Vivo Relationships (IVIVR)}

Product oral bioavailability is a function of several processes including in vivo product dissolution, drug movement across the intestinal membrane, loss due to gastric or intestinal metabolism or to drug instability, intestinal and hepatic first pass metabolism, and presystemic transporter functions. In vitro dissolution methods can only monitor the effect of formulation on in vivo drug dissolution. Thus, while there are a range of potential methods that can be used to establish an in vitro-in vivo relationship (IVIVR) (43), a more precise prediction of in vivo dissolution may best be achieved using in silico physiologically based pharmacokinetic (PBPK) models (44).

Irrespective of the method employed, the underlying assumptions associated with an IVIVR are that formulation is a critical determinant of in vivo oral bioavailability and that there is an in vitro test method that tracks the critical formulation and manufacturing variables influencing in vivo product performance. This complex set of interactions is illustrated in Figure 3.

There are several reasons why an in vitro dissolution method may fail to correctly predict in vivo formulation effects:

- The drug is poorly soluble but very rapidly absorbed. This can promote complete in vivo product dissolution because of physiologically induced maintenance of sink conditions.

- The product being evaluated is a nondisintegrating dosage form (monolithic systems), which may get caught in the stomach where it fails to dissolve.

- The product is a modified-release formulation and the in vitro method fails to adequately capture the rate-limiting process in drug release. This situation reflects a lack of product understanding and failure to identify an appropriate in vitro method.

- There is a specific absorption window in the intestine that, if the product fails to dissolve before passing this point, will result in in vivo failure. In situations where the in vitro method fails to capture these early time-point differences in drug release (too long a delay before first sample), or when the drug goes in solution more rapidly in vitro than in vivo (or vice 
versa), an interpretation of the biological relevance of the in vitro dissolution data can be flawed.

- The selected dissolution test conditions may fail to predict in vivo dissolution. Examples include the failure to use biorelevant media (leading to underor over-estimation of drug release) and excessive agitation (artificial minimization of unstirred boundary layer). The risk of these potential sources of bias can be minimized by drug and drug product understanding as it pertains to the targeted patient population (or animal species) being considered. Excessive or inadequate amounts of surfactant and cosolvents in the dissolution medium can also result in an inability to adequately identify the potential for product in vivo inequivalence or it may exaggerate the true difference in product in vivo performance, leading to an inappropriate interpretation of observed in vitro differences that in fact are without in vivo relevance (45). A diagrammatic representation of this point is provided by Patadia et al. (46).

- An additional critical consideration is the potential for excipients to have a biological effect that may not be predicted based on in vitro dissolution studies alone. For this reason, it is essential to consider the formulations being compared, the potential importance of pre-systemic metabolism or transporter activity in determining drug oral bioavailability, and the likelihood for the excipients used to alter transporter and enzyme function. These potential direct biological effects will go undetected when evaluating comparative in vitro drug dissolution profiles. Table 1 summarizes the known potential in vivo drug-excipient interactions $(47,48)$.

With these points in mind, there are numerous examples where in vitro dissolution approaches could identify critical formulation effects on in vivo product performance (49-53). These examples are highly informative in that they illustrate the kinds of questions and issues that can be resolved by appropriately designed in vitro dissolution test procedures.

In vitro dissolution testing can be a powerful tool for addressing a range of issues if drug and drug product understanding are interwoven into method development and analysis. It is our hope that the points covered in this review will be considered as our scientific community strives to optimize use of this tool for effectively supporting the many questions that influence the safe and effective use of oral pharmaceutical dosage forms.

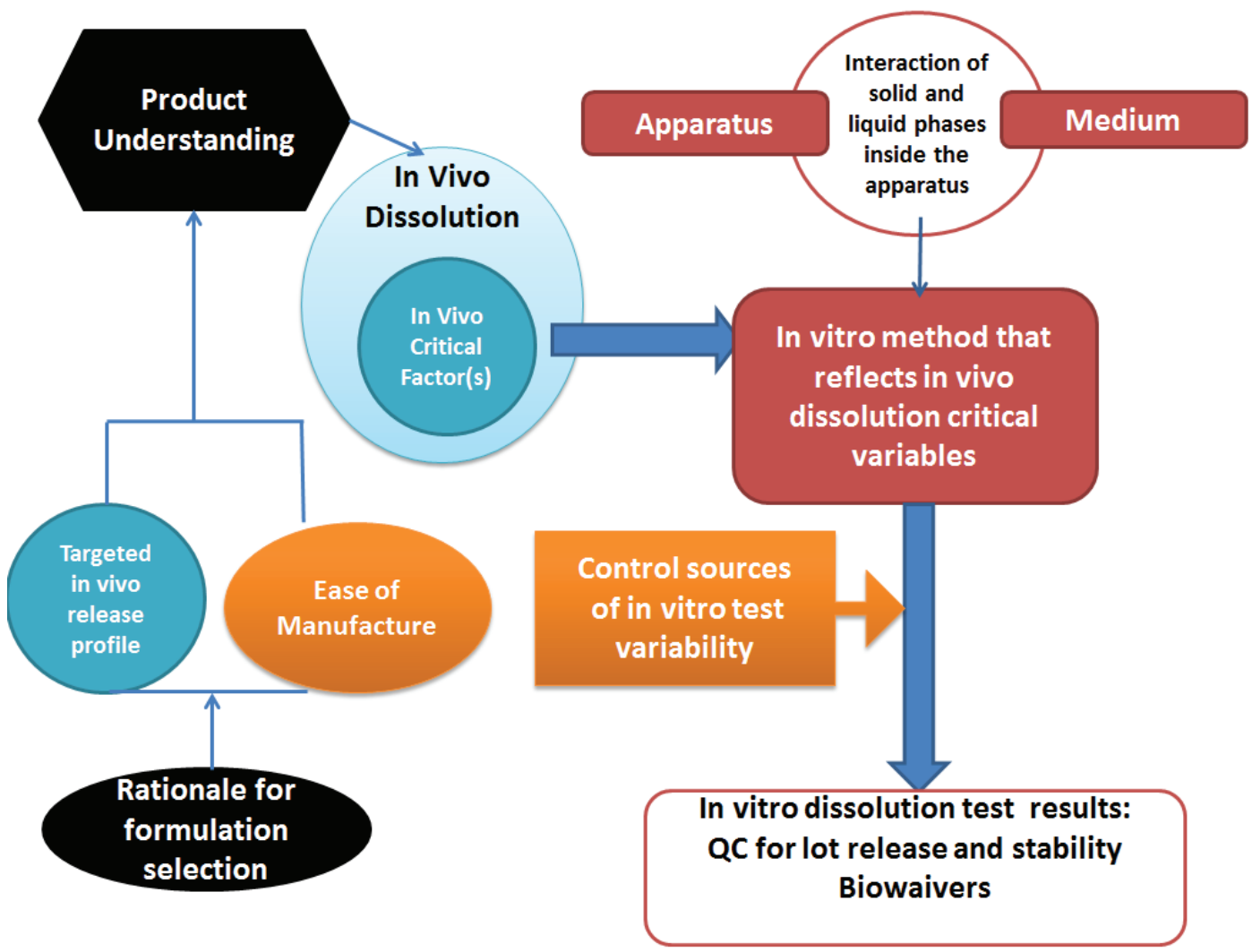

Figure 3. Network of factors that influence in vivo product dissolution, in vitro product dissolution, and the relationship between the two. QC, quality control. 
Table 1. Excipient Effects that can Alter Product Oral Bioavailability through a Biological Effect $(47,48)$

\begin{tabular}{|c|c|c|}
\hline Excipient & System Impacted by Excipient & Effect \\
\hline $\begin{array}{l}\text { Cremophor EL, PEG-40 Stearate, Tween 20, Pluronic F68, } \\
\text { Cremophor RH 40, Tween 80, Solutol HS, Vitamin E TPGS, } \\
\text { Sucrose Laurate, Sodium Deoxycholate, and Dioctyl Sodium } \\
\text { Sulfosuccinate }\end{array}$ & $\begin{array}{l}\text { CYP3A4 (and in some cases, CYP } \\
\text { 2A5) }\end{array}$ & Reduces metabolism \\
\hline $\begin{array}{l}\text { Tween 80, Vitamin E TPGS, Sucrose Laurate, Cremophor EL, } \\
\text { and Cremophor RH 40, Puronic F68 }\end{array}$ & CYP2C9 & Reduces metabolism \\
\hline PEG-40 Stearate & CYP2C19 & Reduces metabolism \\
\hline $\begin{array}{l}\text { Kollicoat Protect, Sodium Caprylate, Vitamin E TPGS, PEG, } \\
\text { AOT }\end{array}$ & CYP2D6 & Reduces metabolism \\
\hline $\begin{array}{l}\text { Tween 20, Cremophor EL, Cremophor RH, PEG 400, Tween } \\
80 \text { and Solutol H15, Propyl Paraben, Methyl Paraben }\end{array}$ & UGT & Varying levels of inhibition \\
\hline $\begin{array}{l}\text { Tween 20, Tween 80, Cremophor EL, Cremophor ELP, } \\
\text { Cremophor RH 40, Cremophor RH 60, and Sodium Lauryl } \\
\text { Sulfate }\end{array}$ & SULT & Varying levels of inhibition \\
\hline $\begin{array}{l}\text { Cremophor EL, Cremophor RH, HPMC, PEG } 40 \text { stearate, } \\
\text { PEG 400, Pluronic F127, F123, and F68, Tween } 20 \text { and } \\
\text { Tween 80, Vitamin E TPGS }\end{array}$ & $\begin{array}{c}\text { P-gp } \\
\text { (efflux transporter) }\end{array}$ & Inhibition \\
\hline $\begin{array}{l}\text { Cremophor EL, Capmul MCM, Cremophor RH 40, Beta- } \\
\text { Cyclodextrin, Labrasol, PEG 2000, PEG 400, Pluronic F127 } \\
\text { and F68, Tween 80, Vitamin E TPGS }\end{array}$ & $\begin{array}{c}\text { MRP2 } \\
\text { (efflux transporter) }\end{array}$ & Inhibition \\
\hline Cremophor EL, Oleic Acid, Pluronic P85, Span 20, Tween 20 & $\begin{array}{c}\text { BCRP } \\
\text { (efflux transporter) }\end{array}$ & Inhibition \\
\hline $\begin{array}{l}\text { Cremophor EL, Hydroxypropryl Beta-Cyclodextrin, PEG 400, } \\
\text { Solutol HS } 15\end{array}$ & $\begin{array}{c}\text { OATP2 } \\
\text { (influx transporter) }\end{array}$ & Inhibition \\
\hline Hydroxypropryl Beta-Cyclodextrin, Solutol HS 15 & $\begin{array}{c}\text { OATP2B1 } \\
\text { (influx transporter) }\end{array}$ & Inhibition \\
\hline Eudragit L100-99 (an enteric coating) & $\begin{array}{c}\text { PEPT1 } \\
\text { (influx transporter) }\end{array}$ & Inhibition \\
\hline Sorbitol and Mannitol & GI transit time & Decrease GI transit time \\
\hline
\end{tabular}

PEG, polyethylene glycol; TPGS, D- $\alpha$-Tocopherol polyethylene glycol 1000 succinate (Vitamin E); CYP, cytochrome P450; AOT, sodium bis-2-ethylhexylsulfosuccinate; UGT, glucuronosyltransferases; SULT, sulfotransferases; HPMC, hypromellose; P-gp, P-glycoprotein; MRP2, multidrug resistance protein 2; $B C R P$, breast cancer resistance protein; OATP, organic anion-transporting polypeptide; PEPTI, peptide transporter 1; GI, gastrointestinal.

\section{SUMMARY}

Of utmost importance is understanding the physicochemical characteristics of the API, the rationale for product formulation selection and for that of the corresponding manufacturing conditions, and whether in vivo dissolution is the rate limiting step in the oral bioavailability process. Second, the dissolution media should have biological relevance. Media volume should be sufficient to maintain sink conditions, although that stipulation may need to be reconsidered for supersaturating formulations. Third, the hydrodynamics of the system can influence the variability associated with the test method. Lastly, the method and the specifications should reflect the stated objective of the test. Different test objectives may necessitate that alternative test conditions be applied.

\section{ACKNOWLEDGEMENT}

The authors wish to express our sincere appreciation to Dr. Gary Hollenbeck for his critical review invaluable suggestions. No funds were allocated or received to support the development of this review.

This article reflects the views of the authors and should not be construed to represent FDA's views or policies.

\section{CONFLICTS OF INTEREST}

The authors declare no conflicts of interested related to this article.

\section{REFERENCES}

1. Ahn, H.; Park, J. H. Liposomal delivery systems for intestinal lymphatic drug transport. Biomater. Res. 2016, 20, 36. DOI: 10.1186/s40824-016-0083-1.

2. Kim, H.; Kim, Y.; Lee, J. Liposomal formulations for enhanced 
lymphatic drug delivery, Asian J. Pharm. Sci. 2013, 8, 96-103. DOI: 10.1016/ajps.2013.07.012.

3. Khan, A. A; Mudassir, J.; Mohtar, N.; Darwis, Y. Advanced drug delivery to the lymphatic system: lipid-based nanoformulations. Int. J. Nanomedicine. 2013, 8, 2733-2744. DOI: 10.2147/IJN. S41521.

4. Wang, T.; Shen, L.; Zhang, Z.; Li, H.; Huang, R.; Zhang Y.; Quan, D. A novel core-shell lipid nanoparticle for improving oral administration of water soluble chemotherapeutic agents: inhibited intestinal hydrolysis and enhanced lymphatic absorption. Drug Deliv. 2017, 24, 1565-1573. DOI: 10.1080/10717544.2017.

5. Rong, W. T.; Lu, Y. P.; Tao, Q.; Guo, M.; Lu, Y.; Ren, Y.; Yu, S. Q. Hydroxypropyl-sulfobutyl- $\beta$-cyclodextrin improves the oral bioavailability of edaravone by modulating drug efflux pump of enterocytes. J. Pharm. Sci. 2014, 103, 730-742. DOI: 10.1002/ jps.23807.

6. Ma, L.; Wei, Y.; Zhou, Y.; Ma, X.; Wu, X. Effects of pluronic F68 and labrasol on the intestinal absorption and pharmacokinetics of rifampicin in rats. Arch. Pharm. Res. 2011, 34, 1939-1943. DOI: 10.1007/s12272-011-1114-z.

7. Mudra, D. R.; Borchardt, R. G. Absorption barriers in the rat intestinal mucosa. 3: Effects of polyethoxylated solubilizing agents on drug permeation and metabolism. J. Pharm. Sci. 2010, 99, 1016-1027. DOI: 10.1002/jps.21836.

8. Koch, K. M.; Parr, A. F.; Tomlinson, J. J.; Sandefer, E. P.; Digenis, G. A.; Donn, K. H.; Powell, J. R. Effect of sodium acid pyrophosphate on ranitidine bioavailability and gastrointestinal transit time. Pharm. Res. 1993, 10, 1027-1030. DOI: 10.1023/A:1018918907670.

9. Adkin, D. A.; Davis, S. S.; Sparrow, R. A.; Huckle, P. D.; Phillips, A. J.; Wilding, I. R. The effects of pharmaceutical excipients on small intestinal transit. Br. J. Clin. Pharmacol. 1995, 39, 381-387. DOI: 10.1111/j.1365-2125.1995.tb04466.x.

10. Buckley, S. T.; Frank, K. J.; Fricker, G.; Brandl, M. Biopharmaceutical classification of poorly soluble drugs with respect to "enabling formulations". Eur. J. Pharm. Sci. 2013, 50, 8-16. DOI: 10.1016/j. eips.2013.04.002.

11. Aungst, B. J. Novel formulation strategies for improving oral bioavailability of drugs with poor membrane permeation or presystemic metabolism. J. Pharm. Sci. 1993, 82, 979-987.

12. <1236> Solubility measurements. In The United States Pharmacopeia and National Formulary USP 41-NF 36; The United States Pharmacoepeial Convention, Inc.: Rockville, MD, 2018.

13. Hörter, D.; Dressman, J. B. Influence of physicochemical properties on dissolution of drugs in the gastrointestinal tract. Adv. Drug Deliv. Rev. 2001, 46, 75-87. DOI: 10.1016/S0169409X(00)00130-7.

14. Takagi, T.; Ramachandran, C.; Bermejo, M.; Yamashita, S.; Yu, L. X.; Amidon, G. L. A provisional biopharmaceutical classification of the top 200 oral drug products in the United States, Great Britain, Spain, and Japan. Mol. Pharm. 2006, 3, 631-643. DOI: 10.1021/mp0600182.

15. Mosharraf, M.; Nyström, C. Apparent solubility of drugs in partially crystalline systems. Drug Dev. Ind. Pharm. 2003, 29, 603-622. DOI: 10.1081/DDC-120021310.

16. Gao, P.; Shi, Y. Characterization of supersaturatable formulations for improved absorption of poorly soluble drugs. AAPS J. 2012, 14, 703-713. DOI: 10.108/s12248-012-9389.

17. Claassen, V. Oral drug administration. In Neglected Factors in Pharmacology and Neuroscience Research; Huston, J. P.; Ed. Elsevier, 1994; pp 59-115.

18. Delaney, J. S. Predicting aqueous solubility from structure. Drug Discov. Today. 2005, 10, 289-295. DOI: 10.1016/s13596446(04)03365-3.

19. Savjani, K. T.; Gajjar, A. K.; Savjan, J. K. Drug solubility: importance and enhancement techniques. ISRN Pharmaceutics. 2012, 195727. DOI: 10:5402/2012/195727.

20. Brouwers, J.; Brewster, M. E.; Augustijns, P. Supersaturating drug delivery systems: the answer to solubility-limited oral bioavailability? J. Pharm. Sci. 2009, 98, 2549-2572. DOI: 10.1002/jps.21650.

21. Laitinen, R.; Löbmann, K.; Grohganz, H.; Priemel, P.; Strachan, C. J.; Rades, T. Supersaturating drug delivery systems: the potential of co-amorphous drug formulations. Int. J. Pharm. 2017, 532, 1-12. DOI: 10.1016/j.ijpharm.2017.08.123.

22. Sun, D. D; Lee, P. I. Haste makes waste: the interplay between dissolution and precipitation of supersaturating formulations. AAPS J. 2015, 17, 1317-1326. DOI: 10.1208/s12248-015-9825-6.

23. Sun, D.D.; Wen, H.; Taylor, L.S. Non-sink dissolution conditions for predicting product quality and in vivo performance of supersaturating drug delivery systems. J. Pharm. Sci. 2016, 105, 2477-2488. DOI: 10.1016/j.xphs.2016.03.024.

24. <1087> Apparent Intrinsic Dissolution - Dissolution Testing Procedures for Rotating Disk and Stationary Disk. In The United States Pharmacopeia and National Formulary USP 41-NF 36; The United States Pharmacopeial Convention, Inc.: Rockville, MD, 2018; pp 7155-7159.

25. Patadia, R.; Vora, C.; Mittal, K.; Mashru, R. Dissolution criticality in developing solid oral formulations: from inception to perception. Crit. Rev. Ther. Drug Carrier Syst. 2013, 30, 495-534.

26. Gao, Z.; Ahadi, S.; Moore, T. W.; Doub, W. H.; Westenberger, B. J.; Buhse, L. F. Effects of vessel geometric irregularity on dissolution test results. J. Pharm. Sci. 2011, 100, 1093-1101. DOI: 10.1002/ jps.22319.

27. Brown, C. K.; Chokshi, H. P.; Nickerson, B.; Reed, R. A.; Rohrs, B. R.; Shah, P. A. Acceptable analytical practices for dissolution testing of poorly soluble compounds. Dissolut. Technol. 2005, 12, 6-12. DOI: 10.14227/DT120405P6.

28. <1092> The Dissolution Procedure: Development and Validation. In The United States Pharmacopeia and National Formulary USP 41-NF 36; The United States Pharmacopeial Convention, Inc.: Rockville, MD, 2018; pp 7178-7198.

29. He, Z.; Zhong, D.; Chen, X.; Liu, X.; Tang, X.; Zhao, L. Development 
of a dissolution medium for nimodipine tablets based on bioavailability evaluation. Eur. J. Pharm. Sci. 2004, 21, 487-491. DOI: 10.1016/j.ejps.2003.11.009.

30. Muertz, A. BioRelevant dissolution media. In Solvent Systems and Their Selection in Pharmaceutics and Biopharmaceutics; Augustijns, P..; Brewster, M. E.; Eds. New York: Springer, 2007; pp 151-177.

31. Galia, E.; Nicolaides, E.; Hörter, D.; Löbenberg, R.; Reppas, C.; Dressman, J. B. Evaluation of various dissolution media for predicting in vivo performance of class I and II drugs. Pharm. Res. 1998, 15, 698-705. DOI: 10.1023/A:1011910801212.

32. Serajuddin, A. T.; Sheen, P. C.; Augustine, M. A. Common ion effect on solubility and dissolution rate of the sodium salt of an organic acid. J. Pharm. Pharmacol. 1987, 39, 587-591. DOI: 10.1111/j.2042-7158.1987.tb03434.x.

33. Corrigan O. I. Co-solvent systems in dissolution testing: theoretical considerations. Drug Devel. Ind. Pharm. 1991, 17, 695-708. DOI: 10.3109/03639049109051600.

34. Dodge, A.; Gould, P. L. Dissolution of chlorpropamide tablets in a methanol-water binary solvent system. Drug Dev. Ind. Pharm. 1987, 13, 1817-1826. DOI: 10.3109/03639048709068694.

35. Dissolution Testing of Immediate Release Solid Oral Dosage Forms; Guidance for Industry; U.S. Department of Health and Human Services, Food and Drug Administration, Center for Drug Evaluation and Research (CDER), U.S. Government Printing Office: Washington, DC, 1997.

36. Diebold, S. Hydrodynamics and dissolution. [Abstract] Dissolut. Technol. 2000, 7, 10. DOI: 10.14227/DT070300P10.

37. Kukura, J.; Arratia, P. E.; Szalai, E.S .; Muzzio, F. J. Engineering tools for understanding the hydrodynamics of dissolution tests. Drug Dev. Ind. Pharm, 2003, 29, 231-239. DOI: 10.1081/DDC120016731.

38. Grady, H.; Elder, D.; Webster, G.K.; Mao, Y.; Lin, Y.; Flanagan, T.; Mann, J.; Blanchard, A.; Cohen, M. J.; Lin, J.; Kesisoglou, F.; Hermans, A.; Abend, A.; Zhang, L.; Curran, D. Industry's view on using quality control, bio relevant, and clinically relevant dissolution tests for pharmaceutical development, registration, and commercialization. J. Pharm. Sci. 2018, 107, 34-41. DOI: 10.1016/j.xphs.2017.

39. Gray. V.; Kelly, G.; Xia, M.; Butler, C.; Thomas, S.; Mayock, S. The science of USP 1 and 2 dissolution: present challenges and future relevance. Pharm. Res. 2009, 26, 1289-1302. DOI: 10.1007/ s11095-008-9822-x.

40. Baxter, J. L.; Kukura, J.; Muzzio, F. J. Shear-induced variability in the United States Pharmacopeia apparatus 2: modifications to the existing system. AAPS J. 2006, 7: 857-864. DOI: 10.1208/ aapsj070483.

41. Beckett, A. N.; Quach, T. T.; Kurs, G .S. Improved hydrodynamics for USP apparatus 2. Dissolut. Technol. 1996, 3, 7-18. DOI: 10.14227/DT030296P7.

42. Mirza, T.; Joshi, Y.; Liu, Q.; Vivlecchia, R. Evaluation of dissolution hydrodynamics in the USP, peak and flat-bottom vessels using different solubility drugs. Dissolut. Technol. 2005, 1, 11-16. DOI: 10.14227/DT120105P11.

43. Nguyen, M. A.; Flanagan, T.; Brewster, M.; Kesisoglou, F.; Beato, S.; Biewenga, J.; Crison, J.; Holm, R.; Li, R.; Mannaert, E.; McAllister, M.; Mueller-Zsgmondy, M.; Muenster, U.; Ojala, K.; Page, S.; Parr, A.; Rossenu, S.; Timmins, P.; Van Peer, A.; Vermeulen, A.; Langguth, P. A. Survey on IVIVC/IVIVR development in the pharmaceutical industry - Past experience and current perspectives. Eur. J. Pharm. Sci. 2017, 102, 1-13. DOI: 10.1016/j. ejps.2017.02.02910.1002/bdd.1844.

44. Otsuka, K.; Shono, Y.; Dressman, J. Coupling biorelevant dissolution methods with physiologically based pharmacokinetic modelling to forecast in-vivo performance of solid oral dosage forms. J. Pharm. Pharmacol. 2013, 65, 937-952. DOI:10.1111/ jphp.12059.

45. Limberg, J.; Potthast, H. Regulatory status on the role of in vitro dissolution testing in quality control and biopharmaceutics in Europe. Biopharm. Drug Dispos. 2013, 34, 247-253. DOI: 10.1002/bdd.1844.

46. Patadia, R.; Vora, C.; Mittal, K.; Mashru, R. Dissolution criticality in developing solid oral formulations: from inception to perception. Crit. Rev. Ther. Drug Carrier Syst. 2013, 30, 495-534. DOI: 10.1615/CritRevTherDrugCarrierSyst.2013007795.

47. Chow, E. C.; Talattof, A.; Tsakalozou, E.; Fan, J.; Zhao, L.; Zhang, $X$. Using physiologically based pharmacokinetic (PBPK) modeling toevaluate the impact of pharmaceutical excipients on oral drug absorption: sensitivity analyses. AAPS J. 2016, 18, 1500-1511. DOI: 10.1208/s12248-016-9964-4.

48. Zhang, W.; Li, Y.; Zou, P.; Wu, M.; Zhang, Z.; Zhang, T. The effects of pharmaceutical excipients on gastrointestinal tract metabolic enzymes and transporters-an ppdate. AAPS J. 2016, 18, 830843. DOI:10-1208/s12248-016-9928-8.

49. Meyer, M. C.; Straughn, A. B.; Jarvi, E. J., Wood, G. C.; Pelsor, F. R.; Shah, V. P. The bioinequivalence of carbamazepine tablets with a history of clinical failures. Pharm. Res. 1992, 9, 1612-1616. DOI: 10.1023/A:1015872626887.

50. Ibarra, M.; Magallanes, L.; Lorier, M.; Vázquez, M.; Fagiolino, P. Sex-by-formulation interaction assessed through a bioequivalence study of efavirenz tablets. Eur. J. Pharm. Sci. 2016, 85, 106-111. DOI: 10.1016/j.ejps.2016.02.001.

51. Bredael, G. M.; Bowers, N.; Boulineau, F.; Hahn, D. In vitro-in vivo correlation strategy applied to an immediate-release solid oral dosage form with a biopharmaceutical classification system IV compound case study. J. Pharm. Sci. 2014, 103, 2125-2130. DOI: 10-1002/jps.24036.

52. Honório Tda, S.; Pinto, E. C.; Rocha, H. V.; Esteves, V. S.; dos Santos, T. C.; Castro, H. C.; Rodrigues, C. R.; de Sousa, V. P.; Cabral, L.M. In vitro-in vivo correlation of efavirenz tablets using GastroPlus ${ }^{\circledR}$. AAPS PharmSciTech. 2013, 14, 1244-1254. DOI: 10.1208/s12249-013-0016-4.

53. Lue, B. M.; Nielsen, F. S.; Magnussen, T.; Schou, H. M.; Kristensen, K.; Jacobsen, L. O.; Müllertz, A. Using biorelevant dissolution to 
obtain IVIVC of solid dosage forms containing a poorly-soluble model compound. Eur. J. Pharm. Biopharm. 2008, 69, 648-657. DOI: 10.1016/.jp2007.

54. Uddina, R.; Saffoon, N.; Sutradhara, K. B. Dissolution and dissolution apparatus: a review. Int. J. Cur. Biomed. Phar. Res. 2011, 1, 201-207.

55. Morihara, M.; Aoyagi, N.; Kaniwa, N.; Katori, N.; Kojim, S. Hydrodynamic flows around tablets in different pharmacopeial dissolution tests. Drug Dev. Ind. Pharm. 2002, 28, 655-662. DOI: 10.1081/DDC-120003856.

56. D'Arcy, D. M.; Corrigan, O. I.; Healy, A. M. Evaluation of hydrodynamics in the basket dissolution apparatus using computational fluid dynamics--dissolution rate implications. Eur. J. Pharm. Sci. 2006, 27, 259-267. DOI: 10.1016/j. ejps.2005.10.007.

57. Hamlin, W.E .; Nelson, E.; Ballard, B. E.; Wagner, J. G. Loss of sensitivity in distinguishing renal differences in dissolution rates due to increasing intensity of agitation. J. Pharm. Sci. 1962, 51, 432-435. DOI: 10.1002/jps.2600510509.

58. Ameur, H.; Bouzit, M. 3D hydrodynamics and shear rates' variability in the United States Pharmacopeia paddle dissolution apparatus. Int. J. Pharm. 2013, 452, 42-51. DOI: 10.1016/ jjharm.201304049.
59. Buckley, S. T.; Frank, K. J.; Fricker, G.; Brandl, M. Biopharmaceutical classification of poorly soluble drugs with respect to "enabling formulations." Eur. J. Pharm. Sci. 2013, 50, 8-16. DOI: 10.1016/j. ejps.2013.04.002.

60. Reddy, L. H.; Murthy, R. S. Lymphatic transport of orally administered drugs. Indian J. Exp. Biol. 2002, 40, 1097-1109.

61. Trevaskis, N. L.; Shackleford, D. M.; Charman, W. N.; Edwards, G. A.; Gardin, A.; Appel-Dingemanse, S.; Kretz, O.; Galli, B.; Porter, C. J. Intestinal lymphatic transport enhances the post-prandial oral bioavailability of a novel cannabinoid receptor agonist via avoidance of first-pass metabolism. Pharm. Res. 2009, 26, 14861495. DOI: 10.007/s11095-009-9860-z.

62. Porter, C. J.; Trevaskis, N. L.; Charman, W. N. Lipids and lipidbased formulations: optimizing the oral delivery of lipophilic drugs. Nat. Rev. Drug Discov. 2007, 6, 231-248. DOI: 10.1038/ nrd2197.

63. Trevaskis, N. L.; Charman, W. N.; Porter, C. J. Lipid-based delivery systems and intestinal lymphatic drug transport: a mechanistic update. Adv. Drug Deliv. Rev. 2008, 60, 702-716. DOI: 10.1016/j. addr.2007.09.007.

\section{Appendix}

\section{COMPENDIAL DISSOLUTION APPARATUSES}

The seven types of standardized USP apparatuses for dissolution are listed below (54).

\section{USP Apparatus 1 (Basket)}

This is a closed system employing a fixed volume. The dosage form is placed inside the basket which revolves at a constant rate. The agitation of this system tends to be less than that associated with USP apparatus 2. As discussed below for the paddle, shaft wobble can influence the reliability and reproducibility of the dissolution results. It reflects a test that is not well controlled. As discussed below for the paddle, the basket also is associated with non-uniform sheer force, particularly when comparing the outer edges versus the center portion of the basket. There also tends to be greater dissolution of the bottom of tablets when placed in a basket as compared to that when using the paddle (55). Furthermore, difficulties can arise due to inadequate mixing at slow speeds, clogging of the mesh openings, ejection of disintegrating particles through the wire basket, lower agitation speeds at the base of the vessel where these escaped particles can accumulate, and an inability to visually observe the disintegration process $(55,56)$.

\section{USP Apparatus 2 (Paddle)}

This is also a closed system with a fixed fluid volume. The paddle has a prescribed size in relation to the vessel containing the fluid and dosage form, and the stirring blades remain at a fixed distance from the sides and bottom of the vessel. The agitation rate (paddle speed) can reduce the thickness of the boundary layer, leading to faster in vitro dissolution. This increase in paddle speed can diminish the ability to distinguish products that will exhibit differences in in vivo dissolution rate (57). In addition, even under normal operating conditions, there are large fluctuations in the fluid velocity such that the sheer rate can vary two- to threefold over the walls and bottom of the vessel. This in turn can result in displacement of the tablets to various locations within the vessel, causing it to be exposed to lower regions of sheer force and therefore greater variability in the dissolution results, this can be minimized using sinkers. Numerous investigations have examined mechanisms for controlling and generating a system with improved (more uniform) hydrodynamics $(58,59)$. As discussed by Baxter et al. (40), this non-uniformity can be visualized as "hot spots" (or regions of high sheer force) within a traditional dissolution vessel. This in turn can influence the reproducibility of the dissolution results. There can also be the problem of coning of the disintegrating dosage form or foaming when surfactants are present in the dissolving medium. These problems can be minimized using an antifoaming agent and by ensuring that tablets are in a position other than the center of the bottom of the vessel. In some cases, the risk of coning 
can be reduced by using peak vessels, which are vessels containing an inverted cone that is molded into the bottom of the vessel (noncompendial). However, the use of peaked vessels can create other hydrodynamic challenges (40). Another point of concern is the potential for system wobble as this too can lead to unequal agitation and therefore a poorly controlled test (note that wobble must also be avoided when using the basket) (58).

\section{USP Apparatus 3 (Reciprocating Cylinder)}

This apparatus consists of an outer and inner vessel. The outer vessel is flat bottomed. The inner is a cylinder that goes up and down in the outer vessel (dips). Because the reciprocating cylinder drains at each upstroke, the cylinder can be transferred to different media, thereby simulating the changing fluid composition encountered as the formulation traverses the GI tract. Advantages of this system are 1) the capability to change the media during the test; 2 ) more turbulent hydrodynamics when compared to USP apparatus 1 and 2; 3) ability to handle products with poorly soluble APIs; and 4) suitability for non-disintegrating tablets and coated granules. The downside of using apparatus 3 is that it does not work well with disintegrating dosage forms and therefore tends to be used primarily with nondisintegrating extended release oral formulations. Other limitations of this equipment are the small volume of the vessel (not more than about $250 \mathrm{~mL}$ ), and foaming with dissolution media containing surfactants due to the more turbulent hydrodynamics (making it necessary to use of antifoam agents).

\section{USP Apparatus 4 (Flow-Through Cell Apparatus)}

This apparatus can be operated as an open or closed system and therefore can be adapted for a wide range of dosage forms. Product dissolution rate is directly proportional to the rate of the fluid flow. This apparatus is appropriate for use across a wide range of dosage forms, but due to the continuous fluid flow, large volumes of fluid may be needed to complete the test.

\section{USP Apparatus 5 (Paddle Over Disk)}

This is a modification of USP apparatus 2 where there is a disk at the bottom of the vessel that serves to constrain the movement of the dosage form. This disk holds the dosage form flat and parallel to the blades. This method is typically used for transdermal system.

\section{USP Apparatus 6 (Cylinder)}

This apparatus consists of a stainless cylinder stirring element, composed of two parts, the upper cylinder and the extension that should be used in accordance with the size of the dosage form. Typically, this is also used to test transdermal system.

\section{USP Apparatus 7 (Reciprocating Holder)}

This is typically used for delivery methods such as osmotic pumps and transdermal systems.

\section{FORMULATION EFFECTS ON IN VIVO DRUG}

\section{ABSORPTION}

Transporter activity can be modulated, either intentionally or unintentionally, by surfactants. For example, numerous nonionic surfactants have been shown to have an inhibitory effect on $A B C$ transporters such as P-glycoprotein (P-gp), breast cancer resistance protein (BCRP) and multidrug resistance proteins (MRPs) (10), thereby increasing the fraction of drug absorbed across the enterocyte membrane. Alternatively, some excipients (e.g., the effect of cyclodextrins as solubilizers or the use of co-solvent systems such as propylene glycol and polyethylene glycol) can simultaneously enhance dissolution but hamper drug permeation (10). These intentional and unintentional formulation effects need to be considered when using in vitro dissolution as a tool to predict in vivo product behavior and subsequent oral bioavailability.

Some formulation strategies influence whether the drug will be absorbed via the hepatic portal vein or by the lymphatics (thereby bypassing first pass drug metabolism) $(60,61)$. For lipophilic compounds (generally, Log $P>5$ ), absorption via the lymphatic system can potentially provide an alternative avenue for systemic access following oral administration of some highly lipophilic drugs. Importantly, this alternative absorption route allows the drug to avoid hepatic first pass metabolism (62). Induction of a fed state can lead to a greater portion of these drugs to be absorbed into the lymphatics, thereby enhancing drug bioavailability (62). Thus, when attempting to enhance drug solubility using lipid based formulations, there is a high likelihood that that formulation strategy will also have other biological consequences that are not detectable via traditional in vitro dissolution methods. An outstanding review of the use of lipids and lipid-based formulations for enhancing drug solubility, permeability, and recruitment of intestinal lymphatic drug transport has been provided by Porter et al. (62) and Trevaskis et al (63). These reviews provide information not only on formulation effects but also on underlying mechanisms by which these formulation effects can occur. 